\section{P059 DISSECTING THE ROLE OF GREMLIN 1 IN SYSTEMIC SCLEROSIS}

L Duffy*, S O'Reilly. Health and Life Sciences, Northumbria University, Newcastle-UponTyne, UK

\subsection{6/annrheumdis-2018-EWRR2019.51}

Career situation of first and presenting author Student for a master or a $\mathrm{PhD}$.

Introduction Systemic Sclerosis (SSc) is a multi-system autoimmune disease, its exact aetiology remains unknown. Despite its heterogeneity, SSc is characterised by three common hallmarks; vascular disruption, production of auto-antibodies and aberrent accumulation of extra cellular matrix (ECM) proteins, leading to fibrosis. Unfortunately, the disease has no cure and few effective therapies. ${ }^{1}$ The aberrent regulation of developmental pathways, including that of bone morphogenetic proteins (BMP) and Gremlin 1, could yield alternative therapuetic targets.

Gremlin 1 is a known BMP antagonist. However, research has found it is implicated in several pathologies including fibrotic diseases such as idiopathic pulmonary fibrosis. $^{2}$ A paper published in 2014 showed that recombinant Gremlin 1 is pro-fibrotic in normal human dermal fibroblasts (NHDF). ${ }^{3}$

Objectives The aim is to investigate the role Gremlin 1 plays in the development of fibrosis in patients with SSc. The key steps to achieving this will involve firstly confirming overexpression of Gremlin 1 leads to an increase in key ECM proteins.

Methods Once confirmed chemical, RNA and clinical antibody interference work will be carried out. Several key molecular techniques will be utilised in the project including transfection, western blotting and reverse transcription polymerase chain reaction.

Results Electroporation was used to transfect NHDF with a Gremlin 1 overexpression vector. Gremlin 1 overexpression showed an increase in Collagen 1 and a statistically significant increase in the myofibroblast marker Alpha Smooth Muscle Actin, compared to control. RNAi work is currently being carried out to asses novel downstream targets. Data suggests cross-talk between the transforming growth factor beta pathway, interaction of Gremlin 1 directly with the vascular endothelial growth factor receptor or a feedback loop involving sonic hedgehog could be involved in the pathway.

Conclusions Due to the rarity, lack of effective therapies and incomplete knowledge of its aetiology research into SSc is required in order to enhance understanding and therapeutic options.

\section{REFERENCES}

1. Kowal-Bielecka O, Landewé R, Avouac J, Chwiesko S, Miniati I, Czirjak L, et al. EULAR recommendations for the treatment of systemic sclerosis: a report from the EULAR Scleroderma Trials and Research group (EUSTAR). Annals of the Rheumatic Diseases 2009;68(5):620-8.

2. Brazil DP, Church RH, Surae S, Godson C, Martin F. BMP signalling: agony and antagony in the family. Trends in Cell Biology 2015;25(5):249-64.

3. O'Reilly S, Ciechomska M, Cant R, van Laar JM. Interleukin-6 (IL-6) trans signaling drives a STAT3-dependent pathway that leads to hyperactive transforming growth factor- $\beta$ (TGF- $\beta$ ) signaling promoting SMAD3 activation and fibrosis via Gremlin protein. Journal of Biological Chemistry 2014;289 (14):9952-60.

Disclosure of Interest None declared.
P062

HISTONE DEACETYLASE 1 (HDAC1): A NOVEL THERAPEUTIC TARGET IN RHEUMATOID ARTHRITIS

${ }^{1} \mathrm{~L}$ Goschl, ${ }^{2} \mathrm{~V}$ Saferding, ${ }^{2} \mathrm{M}$ Kugler, ${ }^{3} \mathrm{~J}$ Backlund, ${ }^{4} \mathrm{P}$ Mathias, ${ }^{2} \mathrm{C}$ Scheinecker, ${ }^{5} \mathrm{~W}$ Ellmeier, ${ }^{2} \mathrm{~S}$ Günter, ${ }^{2} \mathrm{M}$ Bonelli;. 'Division of Rheumatology; ${ }^{2}$ Division of Rheumatology, Medical University of Vienna, Vienna, Austria; ${ }^{3}$ Medical Inflammation Research, Stockholm, Sweden; ${ }^{4}$ Friedrich Miescher Institute for Biomedical Research, Basel, Switzerland; ${ }^{5}$ Division of Immunobiology, Medical University of Vienna, Vienna, Austria

\subsection{6/annrheumdis-2018-EWRR2019.52}

Career situation of first and presenting author Post-doctoral fellow.

Introduction Despite enormous efforts to develop new therapeutic strategies, a large number of patients with Rheumatoid Arthritis (RA) do not sufficiently respond to currently available drugs, which underlies the unmet need to identify new therapeutic targets. $\mathrm{CD}^{+} \mathrm{T}$ cell subsets have been shown to be major drivers of inflammation in RA patients. The expression of their key transcription factors is controlled by histone modifications which includes acetylation of lysine residues mediated by histone deacetylases (HDAC). Pan HDAC inhibitors have been shown to be a potential therapeutic strategy for the treatment of multiple myeloma and cutaneous $\mathrm{T}$ cell lymphoma. However, major side effects limited the clinical use. We therefore addressed the individual role of HDAC1 in a murine arthritis model.

Methods Mice with a $\mathrm{T}$ cell specific deletion of HDAC1 (HDAC1cKO) were generated by using the CD4Cre/LoxP system. Collagen-induced arthritis (CIA) was induced at week 8 . Animals were scored for paw swelling and grip strength. After 10 weeks, mice were sacrificed and paraffin sections of the affected joints were analyzed for histomorphologic signs of inflammation, cartilage and bone destruction. Anti-CII antibody levels were determined by ELISA. To further investigate antibody switch, we performed a DNP-KLH immunization. Serum samples were analyzed for cytokines by multiplex assays. CCR6 expression in $\mathrm{CD}^{+} \mathrm{T}$ cells was analyzed by flow cytometry. Transcriptomic analysis from existing RNA-seq was performed in naïve $\mathrm{T}$ cells and ex vivo isolated Th17 cells from WT mice and STAT3 ${ }^{-/}$mice. Binding of STAT3 to the CCR6 locus was analyzed from previously published STAT3 ChIP-Seq data of IL-6 treated CD4 ${ }^{+}$ T cells.

Results After induction of CIA, HDAC1-cKO mice showed no clinical or histological signs of arthritis. After immunization either with collagen or the model antigen KLH, WT and HDAC1-cKO mice kept regular antibody production, which suggests an undisturbed $\mathrm{T}$ cell $-\mathrm{B}$ cell interaction. The inflammatory cytokines IL-17 and IL-6 were significantly decreased in HDAC1-cKO mice. In addition, CCR6 upregulation was significantly impaired in HDAC1 deficient $\mathrm{CD}^{+} \mathrm{T}$ cells, when cultured in the presence of IL-6. Under these conditions, binding of STAT3 to the CCR6 gene locus was detected. Consecutively CCR6 expression was significantly lower in CD4 T cells isolated from STAT3 ${ }^{--}$mice as compared to WT mice.

Conclusions Our data show the importance of HDAC1 as a key immune regulator in the pathogenesis of $\mathrm{T}$ cell driven collagen induced arthritis. Therefore, it might be considered as an interesting novel therapeutic target in RA.

Disclosure of Interest None declared. 